\title{
Structural and Bonding Trends in Platinum-Carbon Clusters
}

\author{
by \\ THOMAS F. MILLER III \\ 1 \\ Submitted to the \\ Office of Honors Programs and Academic Scholarships \\ Texas A\&M University \\ in partial fulfillment of the requirements for \\ 1998-99 UNIVERSITY UNDERGRADUATE RESEARCH FELLOWS PROGRAM
}

April 15, 1999

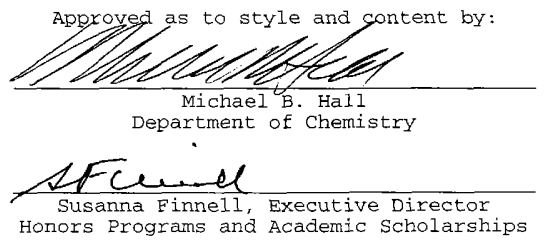

Fellows Group: Chemistry 


\section{Abstract}

\section{Structural and Bonding Trends in Platinum-Carbon clusters \\ Thomas F. Miller III, (Dr. Michael B. Hall) University Undergraduate Fellow, 1998-1999, Texas A\&M University, Department of Chemistry}

Density functional calculations with the B3-LYP functional were used to optimize the platinum-carbon cationic clusters, $\mathrm{PtC}_{\mathrm{x}}{ }^{+}, 1 \leq \mathrm{x} \leq 16$, in both the doublet and quartet states of the linear, fan, open-ring, closed ring, and one-carbon ring geometries. Trends in stability, $\mathrm{Pt}^{+}-\mathrm{C}_{\mathrm{x}}$ binding energy, doublet-quartet excitation energy, and $\mathrm{Pt}-\mathrm{C}$ bond lengths were investigated. Explanations for these patterns are provided in terms of orbital interactions and changes imposed on the carbon-chain by the metal atom. In accord with the previously studied palladium-carbon cations, the $\mathrm{PtC}_{x}{ }^{+}$ clusters favored a linear geometry for $3 \leq x \leq 9$. For larger clusters, the open-ring ( $\mathrm{Pt}$ inserted in $\mathrm{C}_{x}$ ring) and closed ring (Pt bound to two atoms of closed $C_{x}$ ring) families exhibit the lowest energy structures. The stability and the nature of the $\mathrm{Pt}-\mathrm{C}$ bonding in both the closed ring and onecarbon ring ( $\mathrm{Pt}$ bound to one atom of closed $\mathrm{C}_{\mathrm{x}}$ ring) $\mathrm{PtC}_{\mathrm{x}}^{+}$ structures depend greatly on the aromaticity of the corresponding $C_{x}$ ring. However, unlike their palladium counterparts, the closed-ring platinum clusters were found invariably to be more stable than the respective one-carbon species. The stability of forming two Pt-C $\sigma$ bonds is due to relatively lower energy sd hybrid orbitals from the platinum cation. 
TABLE OF CONTENTS

\begin{tabular}{|c|c|}
\hline TABLE OF CONTENTS & 1 \\
\hline LIST OF TABLES & 2 \\
\hline LIST OF FIGURES & 3 \\
\hline INTRODUCTION & 5 \\
\hline THEORETICAL METHODS & 8 \\
\hline SMALL CLUSTERS & 10 \\
\hline LINEAR CLUSTERS & 11 \\
\hline $\mathrm{PtC}^{+}$ & 15 \\
\hline $\mathrm{PtC}_{2 \mathrm{n}+1}{ }^{+}$ & 17 \\
\hline PtC $_{2 \mathrm{n}}{ }^{+}$ & 20 \\
\hline OPEN-RING CLUSTERS & 23 \\
\hline ONE-CARBON AND CLOSED RING CLUSTERS & 28 \\
\hline COMPARISON OF STRUCTURAL FAMILIES & 36 \\
\hline GENERAL CONCLUSIONS & 38 \\
\hline ACKNOWLEDGMENT & 40 \\
\hline REFERENCES & 41 \\
\hline
\end{tabular}


TABLE

Page

I Relative Stablility of the Linear and Fan Structures in the Doublet states

II Pt-C Bond Length in the Doublet and Quartet states of the Linear and open-Ring $\mathrm{PtC}_{\mathrm{x}}{ }^{+}$Clusters

III Energy of the Lone Pair Orbitals in the Doublet State of the Linear $\mathrm{C}_{\mathrm{x}}$ Chains

IV Pt-C Bond Length in the Doublet and Quartet States of the one-Carbon and Closed Ring $\mathrm{PtC}_{\mathrm{x}}{ }^{+}$Clusters

$V$ Mullican Charge of the Platinum Atom in the Doublet state of the One-Carbon Clusters

VI Energy of the Doublet States Relative to the Linear $\mathrm{PtC}_{\mathrm{x}}^{+}$Clusters 


\section{LIST OF FIGURES}

FIGURE

1 The binding energy of the doublet and quartet states of the linear $\mathrm{PtC}_{\mathrm{x}}{ }^{+}$ clusters with respect to $\mathrm{Pt}^{+}$and the corresponding linear $\mathrm{C}_{\mathrm{x}}$ chain.

2 Partial MO diagram showing the orbitals in the frontier region of the linear $\mathrm{C}_{\mathrm{x}}$ chains.

3 Partial Mo diagram showing the interaction of the platinum cation and a single carbon atom.

4 Partial MO diagram showing the interaction of the platinum cation and a linear carbon cluster of even chain length.

5 Partial MO diagram showing the interaction of the platinum cation and a linear carbon cluster of odd chain length.

6 The binding energy of the doublet and quartet states of the open-ring $\mathrm{PtC}_{\mathrm{x}}^{+}$ clusters with respect to $\mathrm{Pt}^{+}$and the corresponding linear $c_{x}$ chain.

7 The frontier orbitals of the linear $C$ clusters upon bending of the chain. (Note that the carbon chain is in the plane of the paper.)

8 Partial MO diagrams showing the interaction of the platinum cation and bent carbon chain in a open-ring cluster. (a) only the $s$ interactions are considered. (b) The $p$ interactions are added to complete the diagram.

9 The binding energy of the doublet and quartet states of the one-carbon $\mathrm{PtC}_{\mathrm{x}}{ }^{+}$ clusters with respect to $\mathrm{Pt}^{+}$and the corresponding $\mathrm{C}_{\mathrm{x}}$ ring.

10 The binding energy of the doublet and quartet states of the closed ring 
$\mathrm{PtC}_{\mathrm{x}}{ }^{+}$clusters with respect to $\mathrm{Pt}^{*}$ and the corresponding $C_{x}$ ring.

11 Spin density localized to each atom in the $\mathrm{PtC}_{\mathrm{x}}^{+}$one-carbon clusters, $6 \leq \mathrm{x} \leq 9$.

12 Partial Mo diagrams showing the interaction of the platinum cation and carbon ring in a one-carbon cluster. 
Introduction

Since the much heralded discovery of buckminsterfullerene in $1985^{1,2}$, there has been considerable interest in both neutral and ionic small carbon clusters. Not only are these clusters likely to serve as precursors to larger fullerene complexes ${ }^{3-7}$, but their manageable size makes them suitable for high-precision theoretical and experimental analysis ${ }^{7-14}$. Although the electronic complexity of these $C_{x}$ clusters has long hindered the conclusive identification of their structural trends, the recent utilization of advanced theoretical techniques has led to significant progress ${ }^{15-25}$. Controversies have arisen regarding the sensitivity of predicted geometries to the method of calculation ${ }^{19-26}$, but it is generally accepted that monocyclic ring conformations are favorable to linear conformations for $x>9^{7,17,21,23,24}$. In addition, evidence exists to suggest that for clusters with $x>14$, both the linear structures and monocyclic rings are suspected to transform from the cumulene-like geometries (equal $\mathrm{C}-\mathrm{C}$ bond lengths) expected for fewer carbon atoms to those more akin to polyacetylenes (significant bond length alternations) $17,20,22,23$.

More recently, the ion-mobility studies of Jarrold ${ }^{27-30}$ have identified the binding of a single transition metal cation to a variety of carbon clusters. However, the nature of this interaction is still unclear because only a very qualitative picture of a compound's structural properties can be derived from ion-mobility experiments, By measuring the 
rate at which a molecule proceeds through an inert gas-filled drift tube, ion-mobility provides the effective collisional cross-section of the molecules in question. This collisional cross-section only indicates the compactness of a complex's geometry, neglecting its more subtle qualities ${ }^{31,32}$. Several theoretical investigations have succeeded in clarifying the structural conclusions that can be drawn from the experimental work $\mathrm{k}^{33-35}$. These studies have shown that for similar complexes, a few basic structural families encompass the most stable geometries for $x \leq 16$ :

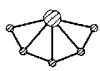

fan

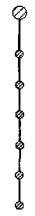

linear

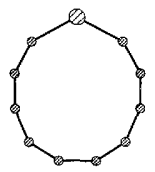

open-ring

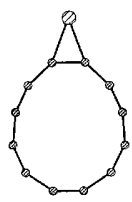

closed ring

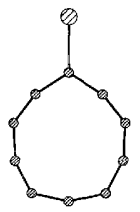

one-carbon ring

However, little is known about the transition metal-carbon bonding or the molecular electronic patterns exhibited by these clusters.

We have calculated the energies, orbital occupations, and optimal geometries of the doublet and quartet states of $\mathrm{PtC}_{\mathrm{x}}{ }^{+}, 1 \leq \mathrm{x} \leq 16$. Among those quantities investigated will be stability, $\mathrm{Pt}^{+}-\mathrm{C}_{x}$ binding energy, doublet-quartet excitation energy, and trends in Pt-C bond lengths. In addition, explanations for these phenomena will be provided from 
analysis of the orbital interactions and changes imposed on the carbon-chain by the metal atom. 


\section{Theoretical Methods}

All calculations in this work were performed with the Gaussian 94 program ${ }^{36}$. Geometry optimizations and energy calculations were carried out using the B3-LYP density functional method, which includes the Becke ${ }^{37}$ three-parameter (B3) exchange functional and the Lee-Yang-Parr ${ }^{38}$ (LYP) correlation functional. All calculations included the effective core potential of Hay and Wadt ${ }^{39}$ for the platinum atoms. The basis set used was the Huzinaga-Dunning ${ }^{39}$ double- $\zeta$ basis set for the carbon atoms, and the double- $\zeta$ basis set of Hay and wadt ${ }^{42}$ for the platinum atoms. In the platinum basis, the two outermost p-functions were replaced by a (41) split of the optimized outer p-function from Couty and Ha $11^{41}$.

As discussed by Plattner et al. ${ }^{20}$, a number of density functional theory (DFT) Eunctionals (viz. B-LYP, B3-LYP, and LSDA) tend to favor cumulene-like geometries for large $C_{x}$ clusters, contrary to the tendency of Hartree-Fock (HF) theory $^{42}$ to prefer localized, polyacetylene-like configurations. However, the utilization of a method containing electron correlation improves the geometric results ${ }^{23.25}$, and DFT more closely reproduces the results obtained from post-HF methods such as $\operatorname{CCSD}(\mathrm{T})$ for small $\mathrm{C}_{\mathrm{x}}$ clusters ${ }^{24}$. Also, the B3-LYP functional is among the most reliable DFT functionals, consistently generating better results than those obtained from both the LDA method and BLYP functional ${ }^{20,25,26,34}$. Thus, it appears safe to assume that 
the B3-LYP functional will give satisfactory results for these complexes and DFT will suffice as the employed method.

Another possible concern is the use of the Kohn-Sham orbitals from the DFT calculations to analyze the bonding in the PtC ${ }^{+}$systems. Although Kohn-Shan orbitals are not equivalent to those obtained from a pure wavefunction method, it will be assumed that the differences are minor enough to bear no significance on the qualitative arguments put forth in this study.

In concurrence with the theoretical and experimental analysis of related $M_{x} C_{y}$ species ${ }^{21,43}$, we found that a number of the cyclic $\mathrm{PtC}_{x}{ }^{+}$clusters underwent slight distortions from $\mathrm{C}_{2 \mathrm{v}}$ symmetry, the highest possible symmetry for monocyclic clusters with an externally bound metal atom. However, these slight geometric aberrations did not seen to be accompanied by significant alterations in energy or electronic structure. Thus, as in previous studies ${ }^{33-35}$, all cyclic complexes were optimized and analyzed in the full $\mathrm{C}_{2 \mathrm{v}}$ point group. 


\section{Small clusters}

As seen in Table $I$, the fan structures for $x \geq 3$ were all highly unstable with respect to the linear $\mathrm{PtC}_{\mathrm{x}}^{+}$complexes. A

Table I. Relative Stablility of the Linear and Fan Structures in the Doublet States

\begin{tabular}{cc}
$\begin{array}{c}\text { Carbon } \\
\text { Atoms }\end{array}$ & $\begin{array}{c}\text { (Doublet Linear-Doublet Fan) } \\
\left(\mathrm{kcal} \mathrm{mol}^{-1}\right)\end{array}$ \\
\hline 2 & 3.98 \\
3 & -57.1 \\
4 & -51.5 \\
5 & -83.7 \\
\hline
\end{tabular}

fan-like geometry is found to be competitive only for the case of $\mathrm{x}=2$. The two equivalent $\mathrm{Pt}-\mathrm{C}$ bonds in the $\mathrm{PtC}_{2}^{+}$fan are formed from the interaction of the carbon orbitals with sd hybrid orbitals on the platinum cation. The low energy of the 6s platinum orbital makes possible this hybridization and leads to the stability of the molecule. Larger clusters were investigated in which the platinum atom bridges the first two carbons of an essentially linear $C_{x}$ chain, but all optimized to the totally linear geometry.

These results are in contrast to the calculations ${ }^{33,34}$ for the early transition metal compounds $\mathrm{LaC}_{\mathrm{x}}{ }^{+}$and $\mathrm{YC}_{\mathrm{x}}{ }^{+}$, in which the fan structure was consistently favored. However, with the electron-rich $\mathrm{d}^{9}$ ground state for $\mathrm{Pt}^{*}$, the formation of more than two $\sigma$ bonds would require the utilization of numerous unoccupied orbitals on the small $C_{x}$ moiety. 


\section{Linear clusters}

Figure 1 and Table II display the binding energies and platinum-carbon bond lengths, respectively, for the doublet and quartet states of each calculated linear cluster. The binding energy was determined by subtracting the total energy of each $\mathrm{PtC}_{x}{ }^{+}$system from the calculated energies of the $\mathrm{Pt}^{+}$ cation in the ${ }^{2} \mathrm{D}$ state and the respective ground-state $\mathrm{C}_{\mathrm{x}}$ chain.

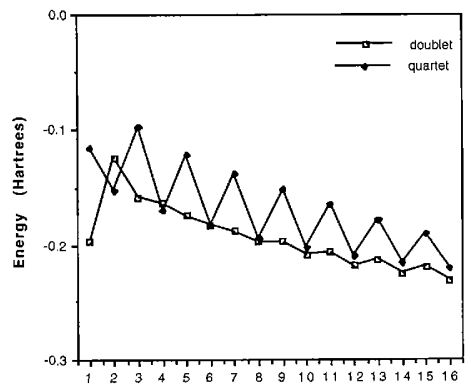

Carbon Atoms

Figure 1. The binding energy of the doublet and quartet states of the linear $\mathrm{PtC}_{\mathrm{x}}^{+}$clusters with respect to $\mathrm{Pt}^{+}$and the corresponding linear $\mathrm{C}_{\mathrm{x}}$ chain.

In general, the $\operatorname{PtC}_{\mathrm{x}}{ }^{+}$systems with even values of $\mathrm{x}$ have energetically competitive doublet and quartet states. For even values of $x$, the doublet states exhibit platinum-carbon bond lengths of $1.76-1.77 \AA$, while the quartet states exhibit considerably longer values at about $1.86 \AA$. In cases of odd 
$\mathrm{x}$, the doublet state is invariably more stable. Also, for odd $x \leq 11$, little difference is found in the platinum-carbon bond lengths of the doublet and quartet states. With larger values of $\mathrm{x}$, however, the bond length in both states shortens dramatically from approximately $1.86 \AA$ to approximately 1.78 $\stackrel{2}{A}$.

Table II. Pt-C Bond Length in the Doublet and Quartet States of the Linear and OpenRing $\mathrm{PtC}_{x}^{+}$Clusters

\begin{tabular}{ccccc}
\hline $\mathrm{x}$ & $\begin{array}{c}\text { Linear } \\
\text { Doublet } \\
(\AA)\end{array}$ & $\begin{array}{c}\text { Linear } \\
\text { Quartet } \\
(\AA)\end{array}$ & $\begin{array}{c}\text { Open-Ring } \\
\text { Doublet } \\
(\AA)\end{array}$ & $\begin{array}{c}\text { Open-Ring } \\
\text { Quartet } \\
(\AA)\end{array}$ \\
\hline 1 & 1.72 & 1.88 & & \\
2 & 1.76 & 1.80 & & \\
3 & 1.84 & 1.84 & & \\
4 & 1.76 & 1.85 & & \\
5 & 1.85 & 1.85 & & \\
6 & 1.76 & 1.86 & 1.87 & 1.93 \\
7 & 1.86 & 1.86 & 1.88 & 1.93 \\
8 & 1.76 & 1.86 & 1.88 & 1.94 \\
9 & 1.86 & 1.86 & 1.90 & 1.88 \\
10 & 1.77 & 1.86 & 1.87 & 1.90 \\
11 & 1.87 & 1.86 & 1.87 & 1.92 \\
12 & 1.77 & 1.87 & 1.87 & 1.93 \\
13 & 1.87 & 1.78 & 1.90 & 1.88 \\
14 & 1.77 & 1.87 & 1.87 & 1.89 \\
15 & 1.77 & 1.78 & 1.87 & 1.91 \\
16 & 1.77 & 1.87 & 1.87 & 1.92 \\
\hline
\end{tabular}

In the quartet state, this contraction of the Pt-C bond occurs at $x=13$, whereas it occurs at $x=15$ in the doublet state. In order to understand these trends we will describe the bonding of the bare $C_{x}$ chains and then the nature of the $\mathrm{Pt}^{+}$interaction with these chains.

First consider the orbitals of the linear $C_{x}$ chains. Our calculations of these carbon clusters are in accord with the results from previous experimental and theoretical 
work ${ }^{17,18,21,22}$. We found ${ }^{1} \Sigma_{g}{ }^{+}$to be the ground state for carbon chains with odd values of $x$, and ${ }^{3} \Sigma_{g}{ }^{-}$to be the ground state for even $x>2$. Our only discrepancy from previous experimenta $1^{3}$ work is for $\mathrm{x}=2$ in which the ${ }^{1} \Sigma_{\mathrm{g}}{ }^{+}$state has been observed to be more stable than the lowest triplet state $\left({ }^{3} \Pi_{u}\right)$ by $716 \mathrm{~cm}^{-1}$. Our calculations found the lowest-energy triplet (unrestricted single determinant) to be favored over the ${ }^{1} \Sigma_{g}{ }^{+}$ state by $6966 \mathrm{~cm}^{-1}$. Previous studies using $\mathrm{DFT}^{21}$ have noted the same problem. Fortunately, this small anomaly exists only for the case of $\mathrm{x}=2$.

Figure 2 is a partial molecular orbital diagram showing the terminal lone pairs and lowest $\pi$ molecular orbitals for $C_{x}, 1 \leq x \leq 6$. For $x \geq 2$, the frontier orbitals of even and odd $x$ have very characteristic properties. For all cases, the frontier orbitals exhibit two filled lone pairs of $\sigma$ symetry $\left(\sigma_{g}\right.$ and $\left.\sigma_{u}\right)$ and two sets of unoccupied $\pi$ orbitals (except for $x=2$, which only has one set). Also, the cases with even $x$ exhibit a degenerate pair of singly-occupied $\pi$ orbitals (alternately $\pi_{g}$ and $\pi_{u}$ ), while the cases with odd $x$ have a set of completely filled $\pi$ orbitals (alternately $\pi_{\mathrm{g}}$ and $\pi_{\mathrm{u}}$ ). 


$$
\mathrm{C}_{1}
$$

$\mathrm{C}_{2}$

$\mathrm{C}_{3}$

$\mathrm{C}_{4}$

$\mathbf{C}_{5}$

$\mathrm{C}_{6}$

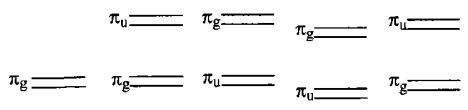

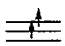
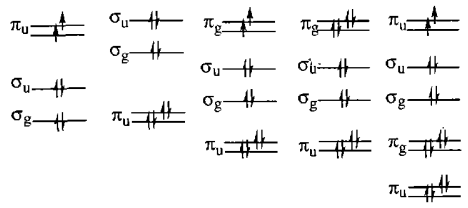

Figure 2. Partial MO diagram showing the orbitals in the frontier region of the linear $C_{x}$ chains.

Figure 2 shows that although the HOMO was of $\sigma$ symmetry for $x=3$, the $C_{5}$ linear cluster has a $\pi$ HOMO. This change in the order of orbital energies with increasing chain length may be described in numerous ways. For the sake of consistency in subsequent explanations, we shall consider the previously existing $\pi$ orbitals to have risen in energy with respect to the $\sigma$ orbitals. Such an occurrence is not at all unexpected. As the carbon chain grows in length, the occupied $\pi$ molecular orbitals will form a continuum or band. That is, increasing numbers of $\pi$ orbitals will be found around the HOMO-LuMo gap, eventually converging at the Fermi level. This phenomenon will manifest itself in Figure 2 by the relative destabilization of pairs of occupied $\pi$ orbitals with respect to the $\sigma$ lone pairs. As another example, 
beginning at $x=12$, a second pair of $\pi$ orbitals (completely occupied) becomes less stable than the $\sigma$ lone pairs for the clusters with even $x$. Thus, at $x=13$, two pairs of totally occupied $\pi$ orbitals are less stable than the $\sigma$ lone pairs. (Recall that the first occurred at $x=5$ for the linear clusters with odd $x$.)

An understanding of the bonding in linear $\mathrm{PtC}_{\mathrm{x}}^{+}$clusters can be obtained by considering the interaction of the $5 \mathrm{~d}$ and 6s orbitals of the metal ion with the prescribed frontier orbitals of the respective $\mathrm{C}_{\mathrm{x}}$ cluster. Figures 3,4, and 5 are partial molecular orbital diagrams of linear $\mathrm{PtC}_{x}{ }^{+}$clusters with $x=1, x=2 n+1$, and $x=2 n$, respectively. In each, the $5 d$ and $6 \mathrm{~s}$ orbitals from $\mathrm{Pt}^{+}$on the left are shown to interact with orbitals from various $C_{x}$ chains on the right. These diagrams will be utilized in the following discussion.

PtC $^{+}$. Consider first the special case of $\mathrm{x}=1$ (Figure 3). A close examination of this diagram is worthwhile because it contains many of the essential elements of the more complicated Figures 4 and 5. Clearly, the $\delta$ orbitals of $\mathrm{Pt}^{+}$are nonbonding because of the lack of available orbitals of the same symmetry on the carbon atom. Three significant bonding interactions are found at energies below that of the $\delta$ orbitals. The $5 \mathrm{~d}$ orbitals from $\mathrm{Pt}^{*}$ and the $2 \mathrm{~s}$ and $2 \mathrm{p}$ orbitals from the carbon atom interact to form a $\sigma$ bond, a pair of $\pi$ bonds, and a carbon lone pair of $\sigma$ symmetry. orbital A, which is found at higher energy than the 
nombonding $\delta$ orbitals, would at first glance appear to be strongly antibonding. However, we will see that its strong interaction with the $6 \mathrm{~s}$ orbital from $\mathrm{Pt}^{+}$diminishes this antibonding character.

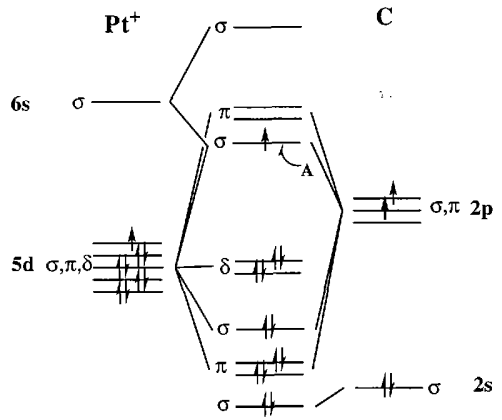

Figure 3. Partial MO diagram showing the interaction of the platinum cation and a single carbon atom.

It is clearly seen from Figure 1 and Table II that the nature of the $\mathrm{Pt}-\mathrm{C}$ bond in the doublet state of $\mathrm{PtC}^{+}$is unique among the complexes examined. Both the reduced bond length and increased binding energy indicate a very strong metalcarbon interaction. Interestingly, the neutral PtC complex, which exhibits the double-occupation of orbital $\mathrm{A}$ in Figure 3. is calculated to have a Pt-C bond length within .003 $\AA$ of that found in $\mathrm{PtC}^{+}$. This constant $\mathrm{Pt}-\mathrm{C}$ bond length implies that orbital $\mathrm{A}$ is essentially nonbonding, allowing both PtC and $\mathrm{PtC}^{+}$to exhibit triple-bond character. The anticipated 
antibonding character in orbital A is essentially eliminated by its stabilizing interaction with the low-lying $\mathrm{Pt}^{+} 6 s$ orbital. Upon excitation to the quartet state, $\mathrm{PtC}^{+}$is expected to promote an electron from one of the nonbonding $\delta$ orbitals to an unoccupied $\pi$ orbital. This transition is calculated to be energetically expensive and occupation of the antibonding orbital leads to an expected stretching of the Pt-C bond (see Table II).

$\mathbf{P t C}_{2 \mathrm{n}+1}{ }^{+}$. Now consider the case of odd values of $x$, $3 \leq x \leq 9$. In Figure 4 , the interactions of the platinum ion with the frontier orbitals of the respective $C_{x}$ chain are shown. For odd $x$, the doublet states are calculated to be considerably more stable than the quartet states. Here, the doublet-quartet transition entails a costly excitation of one of the electrons in the terminal lone pair (orbital $C$ ) of the carbon chain to an unoccupied $\pi$ orbital $E$ of primarily carbon character. Because this transition only involves orbitals on the carbon chain, no change is observed in the Pt-C bond length. 


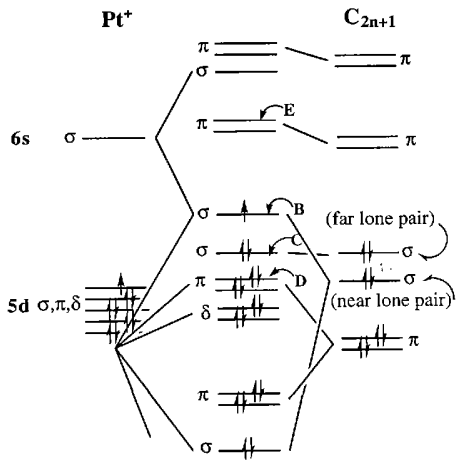

Figure 4. Partial MO diagram showing the interaction of the platinum cation and a linear carbon cluster of odd chain length.

As in the linear $C_{x}$ chains, changes in orbital occupation are found in the $\mathrm{PtC}_{\mathrm{x}}{ }^{+}$clusters with increasing $\mathrm{x}$. It was noted earlier that as the $C_{x}$ chain increases in length, more orbitals of $\pi$ symmetry appear in the frontier region. For odd values of $x$, Figure 4 can be used to explain the approach to the $\pi$ continuum via the gradual rise of orbital $D$ with respect to the localized $\sigma$ orbitals. Also, more $\pi$ orbitals of similar character will fill in below orbital D. At $\mathrm{x}=11$, orbital D becomes less stable than C for the quartet state, changing the orbital occupation in the quartet state and the nature of the doublet-quartet transition. This excitation now involves the promotion of an electron from a Pt-C $\pi$ orbital (orbital D) to a $\pi$ orbital (orbital E) comprised 
primarily of carbon character. Thus, in the quartet state, a carbon lone pair (orbital c) is double-occupied in favor of a $\pi$ antibonding orbital (orbital D). It might initially seem peculiar that this causes no significant change in the Pt-C bond length of the quartet state. However, as orbital D rises in energy relative to the lone pairs, it participates in a relatively smaller portion of the net metal-carbon $\pi$ interaction than it did for shorter carbon chains. In addition, the lowest energy unoccupied $\pi$ orbital will likewise become more non-bonding as it approaches the $\pi$ continuum from above. This is evidenced by the decreasing doublet-quartet splitting energy seen in Figure 1 for odd $x$ and leads to less antibonding character in orbital E. Consequently, the orbital switch at $x=11$ for the quartet state is not accompanied by a shortening of the $\mathrm{Pt}-\mathrm{C}$ bond.

For $x \geq 13$ in the quartet state, orbital $D$ is calculated to also become less stable than orbital B. Thus, a change of electron configuration occurs as an electron is switched from orbital D to orbital B. Primarily due to mixing from the platinum atom's low-lying $6 \mathrm{~s}$ orbital, orbital B exhibits considerable $\sigma$-bonding character. Thus, the change in the orbital occupation at $\mathrm{x}=13$ for the quartet state results in an decrease in Pt-C bond length. This ordering of the frontier orbitals is expected to be maintained for larger values of $x$. For the doublet state, it is not until $x=15$ that orbital $B$ is calculated to be filled in favor of orbital 
D. As above, the double occupation of the $\sigma$-bonding orbital $\mathrm{B}$ leads to a reduction in the $\mathrm{Pt}-\mathrm{C}$ bond distance.

It is clear from their composition that the orbitals $A$ and $B$ from Figures 3 and 4 , respectively, would exhibit very similar characteristics. However, as previously mentioned, orbital B will exhibit considerably more bonding character. It can be seen in Table II that changing $x=1$ to odd $x>1$ leads to a substantial increase in the $\mathrm{Pt}-\mathrm{C}$ bond length. Thus, for the case of odd $x>1$, the antibonding orbital contributions from the carbon lone pair and $\sigma \mathrm{Pt}^{+}$orbitals are less. Orbital $\mathrm{B}$ is less destabilized by the antibonding interaction and has an increased bonding character.

$\mathrm{PtC}_{2 \mathrm{n}}{ }^{+}$. Now consider the binding energy trends in $\mathrm{PtC}_{\mathrm{x}}{ }^{+}$ for the case of even $x, 2 \leq x \leq 16$. Invariably, the doubletquartet transition for these complexes is less energetically costly than for odd $x$. In fact, the quartet is found to be the most stable species until $x \approx 6$. It can be seen in Figure 5 that the transition involves the promotion of an electron from orbital $F$ to orbital $G$. In similar fashion to the previously discussed orbital $B$, orbital $F$ will have notable $\sigma$-bonding character. Also, orbital G will portray negligible $\pi$-antibonding character for larger values of $x$. Thus, the calculated Pt-C bond distances for the quartet state exhibit the expected increase as the metal-carbon bond order is depleted by the promotion of an electron from a bonding orbital (F) to a nonbonding one (G) . 


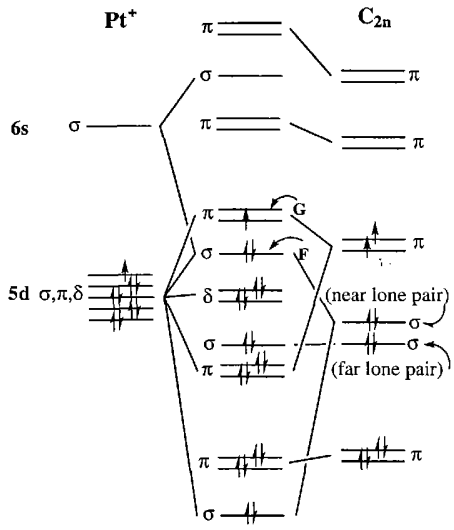

Figure 5. Partial MO diagram showing the interaction of the platinum cation and a linear carbon cluster of even chain length.

Conveniently, the analysis for the $\mathrm{PtC}_{x}{ }^{+}$linear clusters with even $x$ is much less involved than that for the clusters with odd $x$. This is due primarily to the fact that in the calculations performed on the systems with even $x$, no changes in orbital occupations were found. However, for some $x>16$, it is expected that an occupied $\pi$-nonbonding orbital will rise above orbital $F$ in Figure 5, causing a switch in the electron configuration of the quartet states with even $x$. This alteration will be very much akin to those previously examined, leading most likely to a contraction of the Pt-C bond length to about $1.77 \AA$. 
For linear $\mathrm{PtC}_{\mathrm{x}}{ }^{+}$clusters with both even and odd values of $x$, Figure 1 displays a gradual increase in binding energy as $x$ increases. This increasing $P t-C$ bond order for even $x$ is attributed to the rising energy of the $C_{x}$ lone pairs (Table III). It is clearly seen in Figure 5 that as the bonding carbon lone pair (labeled "near lone pair") approaches the 5d $\mathrm{Pt}^{+}$orbitals in energy, a greater $\sigma$ interaction will occur. A Mulliken population analysis of this series also indicates that the most rapidly increasing interaction is between the platinum 5d orbitals and the lone pair of the carbon chain. Consequently, an increase in binding energy would be expected with longer $C_{x}$ chain lengths. An entirely analogous argument holds for the case of odd $x$, explaining the generally downward slope in Figure 1.

Table III. Energy of the Lone Pair Orbitals in the Doublet State of the Linear $\mathrm{C}_{\mathrm{x}}$ Chains

\begin{tabular}{ccc}
\hline $\mathbf{x}$ & $\begin{array}{c}\sigma_{\mathrm{u}} \\
\text { (hartrees) }\end{array}$ & $\begin{array}{c}\sigma_{\mathrm{g}} \\
\text { (hartrees) }\end{array}$ \\
\hline 4 & -.361 & -.347 \\
6 & -.340 & -.338 \\
8 & -.329 & -.329 \\
10 & -.322 & -.321 \\
12 & -.316 & -.316 \\
14 & -.312 & -.311 \\
16 & -.308 & -.308 \\
\hline
\end{tabular}




\section{Open-Ring clusters}

Figure 6 portrays the calculated binding energies for the open-ring clusters, $6 \leq x \leq 16$, relative to the linear $\mathrm{C}_{\mathrm{x}}$ chain and platinum cation. In all cases, the doublet state was found to be more stable that the quartet. However, the two states are energetically competitive for the open-ring $\mathrm{PtC}_{\mathrm{x}}{ }^{+}$clusters with $\mathrm{x}=4 \mathrm{n}+2$. In general, the $\mathrm{Pt}-\mathrm{C}$ bond lengths (Table II) are longer that those calculated for the linear clusters. (Note that each open-ring cluster has two equivalent $\mathrm{Pt}-\mathrm{C}$ bonds.) The metal-carbon bond length remains relatively constant for the doublet state at $1.87-1.90 \AA$, and the Pt-C bond length in the quartet state is calculated to vary from 1.88-1.93 А.

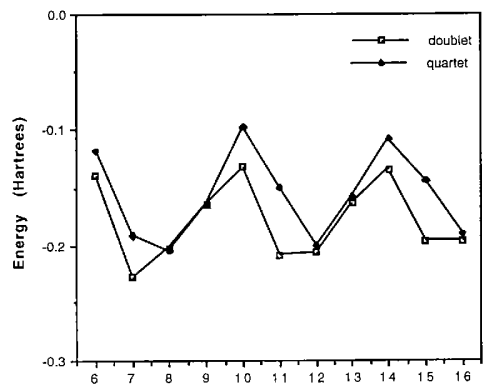

Carbon Atoms

Figure 6. The binding energy of the doublet and quartet states of the open-ring $\mathrm{PtC}_{\mathrm{x}}{ }^{+}$ clusters with respect to $\mathrm{Pt}^{+}$and the corresponding linear $\mathrm{C}_{\mathrm{x}}$ chain. 
The bonding of the linear $C_{x}$ and linear $\operatorname{PtC}_{x}{ }^{*}$ clusters provide deep insight into the electronic structure of the open-ring $\mathrm{PtC}_{\mathrm{x}}{ }^{*}$ clusters. Quite simply, these complexes can be visualized as linear clusters in which the terminal lone pair bends around to interact with the metal atom. A complete bonding description may be obtained by considering the frontier orbitals in the $c_{x}$ clusters distorted to interact in the fashion of the open-ring clusters.

The differences in the bonding of the linear and openring $\mathrm{PtC}_{\mathrm{x}}^{+}$clusters arise from the physical distance between the platinum cation and the endpoints of the respective $C_{x}$ moieties. In the linear clusters, only one end of the carbon chain interacts significantly with $\mathrm{Pt}^{+}$. On the other hand, both ends of the $c_{x}$ chain are close enough in the open-ring clusters to have bonding interactions. Thus, the parity of the orbital contributions from the endpoints of the $C_{x}$ chain is a factor in the open-ring clusters that did not require consideration in the linear clusters. Figure 7 displays the irreducible representations to which the frontier orbitals of the carbon chains correspond upon distortion from the linear geometry. The figure shows that classification of the frontier $\pi$-like orbitals from the carbon chains in $C_{2 v}$ symmetry will divide them into four groups. By analogy with the orbitals which lead to Hueckel's rules of aromaticity, a four-carbon "periodicity" may be expected in the bonding trends of the open-ring clusters. 


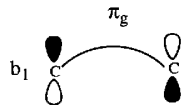

$a_{2}$
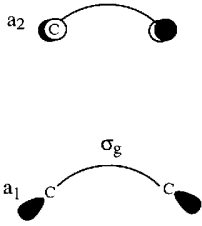

$a_{1}$

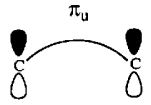

$\mathrm{b}_{2}$

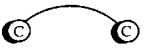

Figure 7. The frontier orbitals of the linear $\mathrm{C}$ clusters upon bending of the chain. (Note that the carbon chain is in the plane of the paper.)

As seen in Figure 6 , the binding energy clearly undulates as expected. The rapid increase in binding energy (with respect to $\mathrm{Pt}^{+}$and the linear $\mathrm{C}_{x}$ chain) with larger $\mathrm{x}$ is primarily due to a reduction in the strain of bending the linear carbon chain to form the open-ring clusters. The long Pt-C bond distances (Table II) are necessarily connected with less $\pi$-bonding in the open-ring clusters. The $\pi$-like orbitals (see Figure 7) will have relativeiy small overlap with the platinum d orbitals. However, it will be shown that the existing $\pi$ bonding leads to interesting trends in the doublet-quartet splitting energies.

A particularly obvious trend seen in Figure 6 is that the doublet-quartet splitting is considerably larger for the $\mathrm{PtC}_{x}{ }^{+}$open-ring clusters in which $\mathrm{x}$ equals $4 \mathrm{n}$ than those in which it equals $4 \mathrm{n}+2$. (Recall that these splittings were essentially identical in the case of the linear isomers.) It 
is seen from Figure 2 that for both $x=4 n$ and $x=4 n+2$, the $C_{x}$ cluster will contribute two fully occupied $\sigma$-like orbitals and two half-filled $\pi$-like orbitals and two unoccupied $\pi$-like orbitals to the frontier orbital region. Figure 7 displays the symmetry of these orbitals after the $C_{x}$ chain has been distorted. The $\sigma$ bonding for the cases of $x=4 n$ and $x=4 n+2$ are essentially identical. "For clarity, the molecular orbital diagram for the open ring clusters will be constructed in two steps. First, the interaction of the orbitals of the metal ion with the lone pairs of the $C_{x}$ clusters can be seen in Figure 8a. One molecular orbital diagram can suffices for $x=4 n$ and $x=4 n+2$ because the symmetry of the o-like orbitals are the same for both cases. Differences in the bonding of the two classes appear as a result of the $\pi$ interactions displayed in Figure $8 \mathrm{~b}$. In the completed molecular orbital diagram (Figure $8 \mathrm{~b}$ ), it can be seen that the $\pi$-like orbitals on the carbon chains are contrasting for $x=4 n$ and $x=4 n+2$. Although both cases exhibit an unoccupied pair and a half-filled pair of orbitals, the symmetries of these pairs are switched. Notice that for the case in which $\mathrm{x}=4 \mathrm{n}$, the excitation to the quartet state will entail promoting an electron from orbital $H$ to orbital $I$, a high energy $\pi$ orbital located mostly on the carbon chain. However, for the case involving $x=4 n+2$, a lower energy excitation is available. orbital $K$, a metallic orbital exhibiting weak $\sigma$ and $\pi$ antibonding character will fall 
considerably lower in energy than the excited orbitals located on the carbon chain. Thus, the energy required to excite an electron from orbital $H$ to orbital $I$ will be decidedly more than that required to excite an electron from orbital $J$ to orbital $\mathrm{K}$. This explains the observed trend in Figure 6.

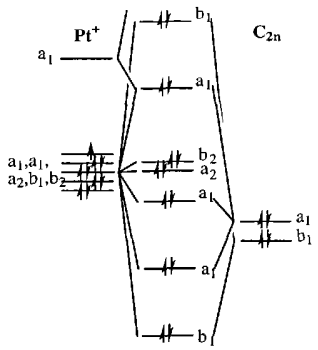

(a)

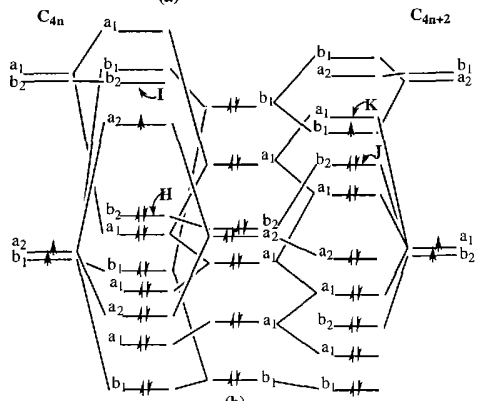

(b)

Figure 8. Partial MO diagrams showing the interaction of the platinum cation and bent carbon chain in a open-ring cluster. (a) Only the $\sigma$ interactions are considered. (b) The $\pi$ interactions are added to complete the diagram. 


\section{One-Carbon and closed-Ring clusters}

The bonding in clusters involving a complete carbon ring will be rationalized primarily in terms of the expected Hueckel orbitals. The $\mathrm{C}_{\mathrm{x}}$ rings each contain two conjugated systems: one in the plane of the molecule and the other perpendicular to this plane. Based on the expectation that these two systems become essentially identical at large $x$, all of the $C_{x}$ rings were examined in a spin state where an equal number of electrons occupy the two conjugated systems.

To best model the $\mathrm{Pt}^{*}-\mathrm{C}_{\mathrm{x}}$ interaction, the geometries of the lone $C_{x}$ rings were first optimized with DFT under two constraints. The first is that they would remain planar. The second constraint was that the rings would display a $\mathrm{C}_{2}$ axis of symmetry coincident with that in the $C_{x}$ moiety in the associated $\mathrm{PtC}_{x}^{+}$species. Unfortunately, this leads to a complication for the $C_{x}$ rings with even $x$. Two nonequivalent $\mathrm{C}_{2}$ axes are possible for each ring:

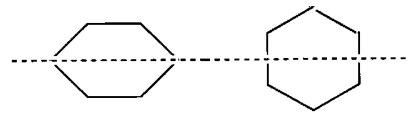

(A)

(B)

These are best visualized in terms of the respective $C_{2}$ axes of the one-carbon and closed-ring $\mathrm{PtC}_{\mathrm{x}}^{+}$clusters. In the cases where nonequivalent $C_{2}$ axes simultaneously exist, we simply calculated both possible $C_{x}$ structures and separately utilized them where appropriate. In general, examination of the one-carbon $\mathrm{PtC}_{\mathrm{x}}{ }^{*}$ clusters with even $\mathrm{x}$ will be with respect 
to (A), whereas (B) will be employed for the corresponding closed-ring structure. At most, we found the energies of (A) and (B) to differ by less than $3.7 \mathrm{kcal} \mathrm{mol}^{-1}$.

The aforementioned assumptions are by no means flawless. For example, we found that the $\mathrm{C}_{7}$ ring favors a triplet spin state with both unpaired electrons in the plane of the molecule. Also, frequency calculations ${ }^{21}$ have shown that the ground-state geometry of many $c_{x}$ rings with even $x$ do not have $\mathrm{C}_{2 \mathrm{v}}$ symmetry. However, deviation from these approximations has been found to be subtle and highly method-dependant ${ }^{23}$. The description of the metal-carbon interaction and the nature of the bonding trends will not be affected.

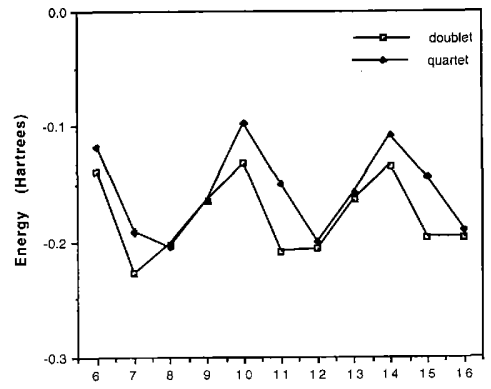

Carbon Atoms

Figure 9. The binding energy of the doublet and quartet states of the one-carbon $\mathrm{PtC}_{\mathrm{x}}^{+}$ clusters with respect to $\mathrm{Pt}^{+}$and the corresponding $\mathrm{C}_{\mathrm{x}}$ ring. 


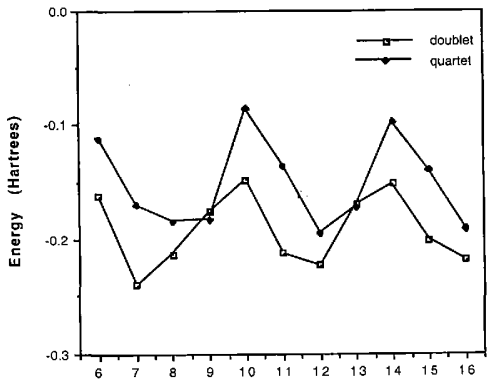

Carbon Atoms

Figure 10. The binding energy of the doublet and quartet states of the closed ring $\mathrm{PtC}_{\mathrm{x}}^{+}$ clusters with respect to $\mathrm{Pt}^{+}$and the corresponding $\mathrm{C}_{\mathrm{x}}$ ring.

Figures 9 and 10 exhibit the binding energy of the onecarbon and closed-ring $\mathrm{PtC}_{x}{ }^{+}$clusters with respect to the $\mathrm{Pt}^{+}$ cation and the $\mathrm{C}_{x}$ carbon ring. The $\mathrm{Pt}-\mathrm{C}$ bond lengths are displayed in Table IV. Both groups of clusters are most weakly bound for the case of $x=4 n+2$, and the doublet-quartet splitting energies are greatest for $x=4 n+2$ and $x=4 n+3$. The binding energies of the doublet states are calculated to increase substantially for the cases of $x=4 n+3$ and $x=4 n$ in both the one-carbon and closed-ring clusters. As seen in Table IV, the length of the Pt-C bond in the one-carbon clusters undergoes dramatic oscillations as the number of carbon atoms is increased. For the doublet state, the Pt-C bond is longest in the one-carbon $\mathrm{PtC}_{\mathrm{x}}{ }^{+}$clusters when $\mathrm{x}=4 \mathrm{n}+2$, 
and it is shortest when $x=4 n$. Conversely, the two equivalent Pt-C bonds in the closed-ring clusters remain essentially constant for all calculated values of $x$ (Table IV). The Pt-C bonds are 1.95-1.99 $\mathrm{A}$ in the doublet states and $1.96-1.98 \AA$ in the quartet states of the closed-ring clusters.

Table IV. Pt-C Bond Length in the Doublet and Quartet States of the One-Carbon and Closed Ring $\mathrm{PtC}_{x}{ }^{+}$Clusters

\begin{tabular}{ccccc}
\hline $\mathrm{x}$ & $\begin{array}{c}\text { One-Carbon } \\
\text { Doublet } \\
(\AA)\end{array}$ & $\begin{array}{c}\text { One-Carbon } \\
\text { Quartet } \\
(\AA)\end{array}$ & $\begin{array}{c}\text { Closed Ring } \\
\text { Doublet } \\
(\AA)\end{array}$ & $\begin{array}{c}\text { Closed Ring } \\
\text { Quartet } \\
(\AA)\end{array}$ \\
\hline 6 & 1.95 & 1.90 & 1.95 & 1.96 \\
7 & 1.88 & 1.96 & 1.96 & 1.97 \\
8 & 1.84 & 1.88 & 1.96 & 1.96 \\
9 & 1.88 & 1.92 & 1.99 & 1.96 \\
10 & 1.97 & 1.91 & 1.96 & 1.97 \\
11 & 1.90 & 1.98 & 1.97 & 1.97 \\
12 & 1.86 & 1.90 & 1.97 & 1.98 \\
13 & 1.90 & 1.93 & 1.99 & 1.97 \\
14 & 1.97 & 1.92 & 1.97 & 1.98 \\
15 & 1.91 & 1.99 & 1.98 & 1.97 \\
16 & 1.87 & 1.91 & 1.98 & 1.98 \\
\hline
\end{tabular}

The periodicity displayed in the Figures 9 and 10 immediately suggests that the aromaticity of the carbon rings is a large factor in the nature of the $\mathrm{Pt}^{+}-\mathrm{C}_{\mathrm{x}}$ bonding. For $x=4 n+2$ (the case of maximum aromaticity), the planar $C_{x}$ moiety would be relatively stable unto itself and have little to gain from an interaction with the platinum cation. For $\mathrm{x}=4 \mathrm{n}$, however, the antiaromatic $c_{x}$ ring would be driven toward the relief afforded by an interaction with the metal. Electron density obtained from the $\pi$-like orbitals of the platinum cation would make the $\mathrm{C}_{x}$ ring more aromatic in character. These hypothesis are supported for the one-carbon clusters by spin density data presented in Figure 11. 

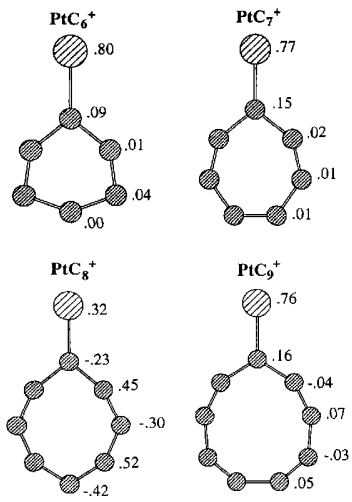

Figure 11. Spin density localized to each atom in the $\mathrm{PtC}_{\mathrm{x}}{ }^{+}$one-carbon clusters, $6 \leq \mathrm{x} \leq 9$.

Note that for the doublet case of $x=6 \quad(x=4 n+2)$, the Pt-C bond distance is $1.95 \AA$ and the radical electron is primarily located on the platinum cation. This indicates a minor interaction between the closed shell $C_{x}$ ring and the radical metal cation. However, a stronger bond is found for $\mathrm{x}=8$ $(x=4 n)$, in which the bond distance contracts to $1.84 \AA$. The spin density suggests that the radical electron is distributed throughout a $\pi$-orbital composed of contributions from both the platinum atom and the carbon ring system. In general, it appears that the platinum cation is behaving as an "electron-sink." That is, the relatively accessible electrons on the metal atom may be utilized to maximize the aromatic character of conjugated system on the $C_{x}$ moiety. 
Table V clearly shows that the platinum atom donates electron density to the carbon ring in this fashion. In general, the Mulliken charge on the platinum atom displays local maxima at $x=4 n+2$ and local minima at $x=4 n$. These conclusions will now be examined from a somewhat more rigorous standpoint.

Table V. Mullican Charge of the Platinum Atom in the Doublet State of the One-Carbon Clusters

\begin{tabular}{cc}
\hline Carbon Atoms & Charge of Pt Atom \\
\hline 6 & 0.725 \\
7 & 0.654 \\
8 & 0.601 \\
9 & 0.720 \\
10 & 0.794 \\
11 & 0.598 \\
12 & 0.524 \\
13 & 0.651 \\
14 & 0.591 \\
15 & 0.554 \\
16 & 0.465 \\
\hline
\end{tabular}

Figure 12 exhibits Mo diagrams of the one-carbon $\mathrm{PtC}_{x}^{+}$ clusters for the cases $x=4 n+2$ and $x=4 n$. Comparison of the two cases provides insight into their relative binding energies. Note that for $\mathrm{x}=4 \mathrm{n}+2$, the high-energy $\pi$ orbitals on the $\mathrm{C}_{\mathrm{x}}$ ring are unable to contribute much to a stabilizing interaction with the orbitals of the platinum cation. In addition, the relatively large doublet-quartet excitation energy observed in Figure 9 is explained by the need to promote an electron into these unstable $\pi$ orbitals. On the other hand, the respective $\pi$ orbitals in the case of $x=4 n$ are considerably lower in energy. These orbitals have been partially occupied by electrons from the carbon chain and now 
readily participate in stabilizing interactions with the metal cation. The relatively short Pt-C bond length for the $\mathrm{x}=4 \mathrm{n}$ series of one-carbon clusters (see Table IV) is directly attributed to the stability obtained from the overlap of the metal and $\mathrm{C}_{\mathrm{x}}$ orbitals. In addition, the doublet-quartet excitation energy now only requires the transfer of an electron among nearly degenerate $\pi$ orbitals. This is seen in Figure 9 to be a low-energy, and sometimes favorable, transition.

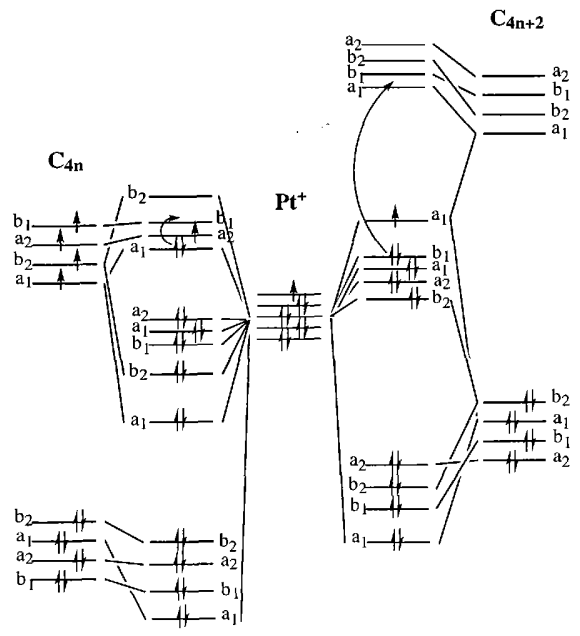

Figure 12. Partial MO diagrams showing the interaction of the platinum cation and carbon ring in a one-carbon cluster. 
Analysis of the closed-ring species yields results that are generally similar to those for the one-carbon clusters above. However, the Pt-C bond length of the closed-ring systems (see Table IV) does not exhibit the same dramatic alternations with increasing $C_{x}$ ring size. This constancy in the bond length is due to fact that the $\mathrm{C}-\mathrm{Pt}-\mathrm{C}$ bond angle is critical to maximizing a favorable orbital interaction. Maintenance of a constant bond angle prevents the closed-ring clusters from displaying the facile bond-length alternations observed in the one-carbon clusters. 


\section{Comparison of structural Families}

In light of the fact that the electronic structures of the closed-ring and one-carbon cluster are so similar, it is interesting to consider which is generally more stable. Table VI indicates that the closed-ring clusters invariably have lower energy. The geometry of the closed-ring systems enables a larger (more $\sigma$-like) interaction between the orbitals of the carbon ring and two of the platinum cation's d orbitals, whereas the one-carbon clusters only exhibit such an interaction for one d orbital. Essentially, the two $\sigma$ bonds of the platinum closed-ring species are stronger than the $\sigma$ bond and $\pi$ bond exhibited by the one-carbon $\mathrm{PtC}_{x}{ }^{*}$ clusters. Such is not invariably the case for the isoelectronic $\mathrm{PdC}_{\mathbf{x}}{ }^{+}$systems ${ }^{35}$ in which the more stable species depends on the value of $x$. As discussed previously for the $\mathrm{PtC}_{2}{ }^{+}$fan structure, the metal component in the $\sigma$ bonds of the closed ring clusters is mostly comprised of sd hybrid orbitals. The greater stability of the closed ring structures is based on the fact that the $6 \mathrm{~s}$ orbital on the metal atom is considerably lower in energy for platinum than for palladium. The lower-energy sd hybrids favor the formation of two $\sigma$ bonds, and, thus, the $\mathrm{PtC}_{x}{ }^{+}$closed-ring species are more readily formed.

As seen for the palladium-carbon cations ${ }^{35}$, the nonlinear $\mathrm{PtC}_{\mathrm{x}}{ }^{+}$systems are favored for $\mathrm{x} \geq 10$ (Table VI). With the increasing number of carbon atoms, the strain of ring formation becomes Iess prohibitive. The open-ring systems, 
which are not subject to the aforementioned aromaticity effects, are calculated to be the most stable species for $x=4 n, x \geq 10$. However, for $x=4 n+2$ and $x \geq 10$, the closed-ring systems are the lowest in energy.

Table VI. Energy of the Doublet States Relative to the Linear $\mathrm{PtC}_{x}^{+}$Clusters

\begin{tabular}{ccccc}
\hline $\mathrm{x}$ & $\begin{array}{c}\text { linear } \\
\left(\mathrm{kcal} \mathrm{mol}^{-1}\right)\end{array}$ & $\begin{array}{c}\text { open-ring } \\
\left(\mathrm{kcal} \mathrm{mol}^{-1}\right)\end{array}$ & $\begin{array}{c}\text { one-carbon } \\
\left(\mathrm{kcal} \mathrm{hol}^{-1}\right)\end{array}$ & $\begin{array}{c}\text { closed ring } \\
\left(\mathrm{kcal} \mathrm{mol}^{-}\right)^{-}\end{array}$ \\
\hline 6 & 0.00 & 21.97 & 50.60 & 38.25 \\
7 & 0.00 & 13.35 & 35.37 & 27.61 \\
8 & 0.00 & 3.19 & 49.22 & 41.91 \\
9 & 0.00 & 9.77 & 51.27 & 45.03 \\
10 & 0.00 & 2.31 & 3.17 & -4.35 \\
11 & 0.00 & -11.18 & -5.32 & -7.67 \\
12 & 0.00 & -15.06 & 11.58 & 1.76 \\
13 & 0.00 & -9.01 & 10.36 & 6.28 \\
14 & 0.00 & -12.67 & -15.59 & -22.54 \\
15 & 0.00 & -25.48 & -23.93 & -26.90 \\
16 & 0.00 & -26.06 & -8.03 & -19.39 \\
\hline
\end{tabular}




\section{General Conclusions}

$A b$ initio quantum chemistry enables the investigation of molecular properties and chemical phenomena from a unique and valuable perspective. Theoretical methods are formed from the application of chemically-motivated constraints to the rigorous theory of quantum mechanics and are rapidly improving in accuracy, consistency, availability, and speed. This development acts not in competition with experimental chemical research, but in a companionship of mutual benefit.

Theoretical chemistry can readily predict information unattainable by experimental means because theory is free of the physical constraints that may hinder an experimental procedure. However, without comparison to reliable experimental results, no theoretical method could ever be effectively evaluated or improved. Theoreticians rely on experimental evidence to rid their methods of pathologies and to create benchmarks of consistency and accuracy.

The marriage of theoreticians and experimentalists is further enriched by the intrinsic link of ab initio methods to our mutual intuition as chemists. A calculation provides explanations for structural and electronic features that are easily translated into the more comprehensible terms of conventional chemistry, precipitating our gradual scientific enlightement. However, the development of chemical understanding is precisely that upon which theory relies to evolve. Quantum chemistry is merely the casting of pure quantum mechanics into the mold of practical chemistry. 
Thus, the success enjoyed by any theoretical model is unalterably determined by the savvy with which that mold was Eormed. 


\section{Acknowledgment}

We thank the National science Foundation (Grant Nos. CHE 94-23271 and CHE 95-28196), the Robert A. Welch Foundation (Grant No. A-648), and the University Undergraduate Research Fellows Program for financial support. 


\section{References}

1) Kroto, H. W.; Heath, J. F.; O'Brien, S. C.; Curl, R. F.; Smalley, R. E. Nature 1985, 318, 162

2) Zhang, Q. L.; O'Brien, S. C.; Heath, J. R.; Liu, Y.; Curl, R. F. Kroto, H. W.; Smalley, R. E. J. Phys. Chem. $1986,90,525$

3) Wang, Z. L.; Kang, Z. C.'J. Phys. Chem. 1996, 100, 17725

4) von Helden, G.; Hsu, M. T.; Gotts, N. ; Bowers, M. T. J. Phys. Chem. 1993, 97, 8182

5) Hunter, J. M. ; FYe, J. L.; Roskamp, E. J.; Jarrold, M. F. J. Phys. Chem. 1994, 98, 1810

6) Hunter, J. M.: Jarrold, M. F. J. An. Chem. Soc. 1995, 117, 10317

7) Weltner, Jr., W.; van Zee, R. J. Chem. Rev. 1989, 89, 1713

8) Taylor, P. R.; Martin, J. M. L; Francois, J. P.; Gijbels, R. J. Phys. Chem. 1991, 95, 6530

9) Watts, J. D.; Bartlett, R. J. J. Chem. Phys. 1992, 96, 6073

10) Sommerfeld, T.; Scheller, M. K., cederbaum, L. S. J. Phys. Chem. 1994, 98, 8914

11) Sowa-Resat, M. B.; Hintz, P. A.; Anderson, S. L. J. Phys. Chem. 1995, 99, 10736

12) Forney, D.; Grutter, M.; Freivogel, P.; Maier, J. P. J. Phys. Chem. 1997, 101, 5292

13) Wang, X. B.; Ding, C. F.; Wang, L. S. J. Phys. Chem. $1997,101,7699$ 
14) Szczepanski, J.; Auerbach, E.; Vala, M. J. Phys. Chem. $1997,101,9296$

15) Martin, J. M. L.; Taylor, P. R. J. Phys Chem. 1996, 100, 6047

16) Watts, J. D.; Bartlett, R. J.; J. Chem. Phys. 1992, 97, 3445

17) Liang, C.; Schaefer, III, H. F.; J. Chem. Phys. 1990, 93, 8844

18) Parasuk, V.; Almlof, J. J. Chem. Phys. 1989, 91, 1137

19) Parasuk, V.; Almlof, J.; Feyereisen, M. W. J. Am. Chem. Soc, 1991, 113, 1049

20) Plattner, D. A.; Houk, K. N. J. Am. Chem. Soc. 1995, 117,4405

21) Hutter, J.; Luthi, H. P.; Diederich, F. J. Am. Chem. SoC. $1994,116,750$

22) Liang, C; Schaefer, III, H. F.; Chem. Phys. Lett. 1990, 169,150

23) Watts, J. D.; Bartlett, R. J. Chem. Phys. Lett. 1992, 190, 19

24) Martin, J. M. L.; Taylor, P. R.; J. Phys. Chem. 1996 , 100,6047

25) Grossman, J. C.; Mitas, L.; Raghavachari, K. Phys. Rev. Lett. 1995, 75, 3870 .

26) Raghavachari, K.; Strout, D. L.; Odom, G. K.; Scuseria, G. E.; Pople, J. A.; Johnson, B. G.; Gill, P. M. W. Chem. Phys. Lett. 1993, 214, 357 
27) Shelimov, K. B; Jarrold, M. F. J. Phys. Chem. 1995, 99, 17677

28) Shelimov, K. B.; Clemmer, D. E.; Jarrold, M. F. J. Phys, Chem. 1995, 99, 11376

29) Clemmer, D. E.; Jarrold, M. F. J. Am. Chem. Soc. 1995, 117, 8841

30) Shelimov, K. B.; Jarrold, M. F. J. Am. Chem. SoC. 1996, 118, 1139

31) Strout, D. L.; Book, L. D.; Millam, J. M.; Xu, C.; Scuseria, G. E. J. Phys. Chem, 1994, 98, 8622

32) Book, L. D.; Millam, J. M.; Xu, C.; Scuseria, G. E. Chem. Phys. Lett. 1994, 222, 281

33) Strout, D. L.; Hall, M. B. J. Phys. Chem. 1996, 100, 18007

34) Strout, D. L.; Hall, M. B. J. Phys. Chem. A 1998, 102, 641

35) Strout, D. I.; Miller III, T. F.; Hall, M. B. J. Phys. Chem. A 1998, 102, 6307

36) Gaussian 94, Revision D.4, M. J. Frisch, G. W. Trucks, H. B. Schlegel, P. M. W. Gill, B. G. Johnson, M. A. Robb, J. R. Cheeseman, T. Keith, G. A. Petersson, J. A. Montgomery, K. Raghavachari, M. A. Al-Laham, V. G. Zakrzewski, J. V. Ortiz, J.B. Foresman, J. Cioslowski, B. B. Stefanov, A. Nanayakkara, M. Challacombe, C. Y. Peng, P. Y. Ayala, W. Chen, M. W. Wong, J. L. Andres, E. S. Replogle, R. Gomperts, R. L. Martin, D. J. Fox, J. S. Binkley, D. J. Defrees, J. Baker, J. P. Stewart, M. 
Head-Gordon, C. Gonzalez, and J. A. Pople, Gaussian, Inc., Pittsburgh PA, 1995.

37) Becke, A. D. J. Chem. Phys. 1993, 98, 5648.

38) Lee, C.; Yang, W.; Parr, R. G. Phys. Rev. B 1988, 37 , 785 .

39) Dunning, T. H.; Hay, P. J. Modern Theoretical chemistry, Ed. H. F. Schaefer, III, 'Plenum; New York, 1976, 1-28.

40) Hay, P. J.; Wadt, W. R. J. Chem. Phys. 1985, 82, 299.

41) Couty, M.; Hall, M. B. J. Comput. Chem. 1996, 17, 1359.

42) Roothaan, C. C. Rev. Mod. Phys. 1951, 23, 69

43) Rubin, Y.; Knobler, C. B.; Diederich, F. J. Am. Chem. SoC. $1990,112,4966$ 